Journal of Bangladesh Chemical Society, Vol. 24(2), 123-134, 2011.

DOI: 10.3329/jbcs.v24i2.9701

\title{
SYNTHESIS OF N-(2'-IMIDAZOLYL)-2- AMINOPYRIDYL PALLADIUM DICHLORIDE AND ITS APPLICATION AS CATALYST IN SUZUKI-MIYAURA CROSS COUPLING REACTION.
}

MD. ABUL HASHEM* AND ABEDA SULTANA

Department of Chemistry, Jahangirnagar University, Savar, Dhaka-1342, Bangladesh

\begin{abstract}
The ligand $\mathrm{N}$-(2'-imidazolyl)-2- aminopyridine 9 was prepared from imidazole. Compound 9 forms complex with $\mathrm{Na}_{2} \mathrm{PdCl}_{4}$ in methanol. The complex $\mathbf{1 0}(1.5 \mathrm{mmol} \%)$ was utilized in Suzuki-Miyaura cross-coupling reaction to prepare substituted biphenyls, terphenyls, and heterobiaryls in good to excellent yields.
\end{abstract}

Keywords: N-(2'-imidazolyl)-2-aminopyridine ligand/Palladium Complexes/ Palladacycles/ Suzuki-Miyaura Cross Coupling/Biaryls/Terphenyls/Heterobiaryls/Suzuki-reaction.

\section{Introduction}

The last half-century has witnessed enormous growth in transition metal- organometalic chemistry, both as scientific discipline and as a subject for research and applications in industry. Organopalladium compounds have a very rich chemistry and are among the readily available and easily prepared and handled of the plethora of known transitionmetal complexes. The facile redox interchange between the two stables Pd (11)/Pd (0) oxidation states is mainly responsible for the rich chemistry enjoyed by palladium compounds. Palladacycles have various activities in the field of Catalysis ${ }^{1-7}$, Medicinal and Biological Chemistry ${ }^{8-9}$, Liquid Crystals (Mesogenic Palladacycles) ${ }^{10}$ and as Photo luminescent material ${ }^{11}$

Recent advances in metal catalyzed coupling methods have allowed increasing usage of cross-coupling reactions in synthetic organic chemistry, for example, the Suzuki-Miyaura $(\mathrm{SM})^{12}$ cross-coupling reaction. Transition-metal-catalyzed cross-coupling reactions have had a major impact on the construction of C-C bonds in functionalized biaryl and heterobiaryl system ${ }^{13}$.

Although many different types of ligands have been discovered for these types of reactions, there is still a need for creative innovations. For example, the development of catalysts not including phosphane ligands is of special interest due to environmental concerns. As reported in several scientific papers, it is very difficult to separate the remaining phosphane moiety during work-up process ${ }^{14}$. Moreover, many ligands are often air-sensitive or expensive, which places significant limits on their synthetic applications.

In recent years, palladacycles as catalyst in cross-coupling reactions have been

*Author to whom all correspondence to be made.E-mail: mdabulhashem@yahoo.com. 
described ${ }^{15}$. There are also homogeneous catalysts, which are reusable, very stable and their enhanced activity has been reported ${ }^{16}$. The complexes of palladium (II) salts with the dipyridine-based ligands of type $\mathbf{1}$, have shown to be efficient catalysts for $\mathrm{C}-\mathrm{C}$ bond forming reactions ${ }^{17}$. Recently, we have reported a new pyridine oxime ligands-based palladium catalyst 2 , for the C-C coupling reactions ${ }^{18}$. ( $\beta$-Oxoiminato)(phosphanyl) palladium complexes ${ }^{19} \mathbf{3}$ have been found to be very active as catalyst in Suzuki-Miyaura coupling reactions. Recently, A. Kirschning and others ${ }^{20}$ have developed a number of new palladacycles (complex $4 \& 5$ ). The dipyridyl-palladium complexes $6 \& 7$, which are easily prepared in good yield and with high purity promote the Suzuki-Miyaura crosscoupling reaction in the preparation of substituted biphenyls, terphenyls, heterobiaryls in good to excellent yields ${ }^{21}$.

In continuation of our work on palladacycles we synthesized more new palladacyclic catalysts based on the derivatives of primary and secondary heteroaromatic amines and the halogen substituted imidazole that can promote carbon-carbon, carbon- nitrogen bond formation. For this purpose we report a newpalladacyclic catalyst $\mathbf{1 0}$ based on the derivative secondary heteroaromatic amine. Our future aim will be to test the biological activity of prepared new palladacyclic catalyst.<smiles></smiles><smiles>CC(C)C1C=CCC1C(C)C</smiles>

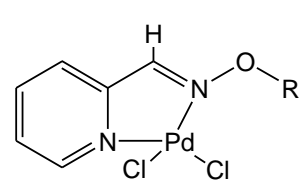

$\mathrm{R}=\mathrm{H}, \mathrm{PhCH}_{2}$,

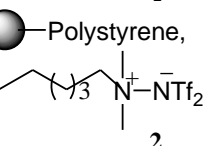<smiles></smiles><smiles>[R]=CCC(CC)Cc1ccc(OC)cc1OC</smiles>

3

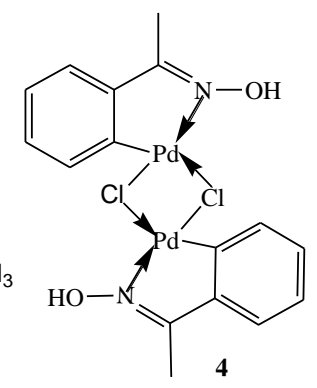<smiles></smiles>

7<smiles></smiles> 
Scheme 1 


\section{Experimental}

${ }^{1} \mathrm{H}-\mathrm{NMR} \&{ }^{13} \mathrm{C}$-NMR Spectra of some samples were recorded using a Bruker WH $400 \mathrm{MHz}$ Spectrometer in the Bangladesh Council of Scientific and Industrial Research (BCSIR) Laboratories, Dhaka and by using $300 \mathrm{MHz}$ NMR-Spectrometer in Leibniz Institute of Plant Biochemistry, Weinberg-3, D-06120 Halle (Saale), Germany and also by using $400 \mathrm{MHz}$ NMR-Spectrometer in Academia Sinica, Nankang, Taipei, Taiwan, Rebublic of China. The spectra were recorded in $\mathrm{CD}_{3} \mathrm{OD}$, DMSO and mostly in $\mathrm{CDCl}_{3}$ with TMS as internal standard. The chemical shifts were expressed in $\delta$-values. Mass spectra were recorded on LC-ESI-MS system in Leibniz Institute of Plant Biochemistry, Weinberg-3, D-06120 Halle (Saale), Germany. The thin layer chromatography (TLC) was performed on precoated aluminium plates (Merck $60 \mathrm{~F}_{254}$ silica gel) and detected by uv light and in Iodine chamber. Column chromatography was performed with Merck silica gel 60, 70-230 mesh ASTM. Yields of the products are the isolated yield after column chromatography. All solvents were dried and purified by usual techniques.

\section{Preparation of 2-Bromoimidazole, 8}

Imidazole $(30 \mathrm{mmol})$ was treated with NBS $(30 \mathrm{mmol})$ in dichloromethane $(10 \mathrm{~mL})$ in presence of silica gel $(2.40 \mathrm{~g})$ under nitrogen at room temperature for 2 hours. After reaction, the product was taken in $10 \mathrm{~mL} \mathrm{MeOH}$ and it was filtered to remove silica gel. The methanolic solution was then evaporated in rotary evaporator to get the solid product. The crude was washed twice with $10 \mathrm{~mL}$ distilled water. After drying in desicator it gave pure crystals. An ash grey colored crystal of 2-bromoimidazole was obtained after removal of solvent.

\section{Preparation of the ligand, $N$-(2'-imidazolyl)-2- aminopyridine, 9}

A reaction of 2-bromoimidazole (6 mmol), 2-aminopyridine (1.410 g $\approx 15 \mathrm{mmol})$, and cesium carbonate $(5.09 \mathrm{~g})$, palladium catalyst, $6(10 \mathrm{mmol})$ in dioxane $(40 \mathrm{~mL})$ was carried out at $100^{\circ} \mathrm{C}$ for 25 hours. Then the reaction mixture was shaken with water (20 $\mathrm{mL})$ and ethyl acetate $(10 \mathrm{~mL})$ and filtered to remove catalyst. Organic layer was collected by extraction with ethyl acetate $(20 \mathrm{~mL})$ and dried over anhydrous magnesium sulfate over night. A red solid was obtained on removal of the solvent in rotary evaporator.

The crude product was chromatographed over a column of silica gel 60 (70-230 mesh) using EA: $\mathrm{MeOH}(9.8: 0.2)$ as eluent. The eluting solvent was collected in several test tubes and the fractions having the same TLC behavior were combined. Compound 9 was obtained as a red solid having melting point $79-80^{\circ} \mathrm{C}$.

General procedure for the preparation of $N$-(2'-imidazolyl)-2-aminopyridyl palladium dichloride complex 10

$\mathrm{N}$-(2'-imidazolyl)-2-aminopyridine, 9 (3-6 mmol) and $\mathrm{Na}_{2} \mathrm{PdCl}_{4}(3.2-6.3 \mathrm{mmol})$ were dissolved in methanol and stirred for 16 hours at room temperature. The solids formed 
were filtered in a sintered crucible. The solids were further washed with methanol to obtain a orange-yellow solid. The complex 10 was dried over conc. $\mathrm{H}_{2} \mathrm{SO}_{4} / \mathrm{P}_{2} \mathrm{O}_{5}$ in a vacuum desicator until constant weight.

\section{General procedure of Suzuki-Miyaura cross-coupling with the complex 10}

Arylboronic acid $(1.5$ mmole $)$, Arylhalide $(1.0 \mathrm{mmole})$, base, $\mathrm{K}_{2} \mathrm{CO}_{3}(2 \mathrm{mmole})$ and toluene (3-4 mL) were mixed together. The mixture was refluxed at $105^{\circ} \mathrm{C}$ for $2-4$ hours. The mixture was cooled to room temperature, then $\mathrm{H}_{2} \mathrm{O}(10 \mathrm{~mL})$ and ethyl acetate $(10$ $\mathrm{mL}$ ) were added; any residual solids present were filtered. To the filtrate ethyl acetate was added again and shaken well. The two layers were separated. The aqueous layer was again extracted with either ethyl acetate or dichloromethane and combined with the original organic layer. The organic phase was dried over anhydrous sodium sulphate. The solvent was removed in rotary evaporator. The crude product was column chromatographed over a silica gel eluting with petroleum ether-ethyl acetate solvent mixture to obtain pure product. Yields were calculated from this isolated products.

Table-1: Spectral data for compounds 8,9 and 10

\begin{tabular}{|c|c|c|c|}
\hline $\begin{array}{l}\text { Compoun } \\
\text { d }\end{array}$ & $\begin{array}{l}{ }^{1} \mathrm{H}-\mathrm{NMR} \\
(\delta, \mathrm{ppm})\end{array}$ & $\begin{array}{l}{ }^{13} \mathrm{C}-\mathrm{NMR} \\
(\delta, \mathrm{ppm})\end{array}$ & $\begin{array}{c}\text { MASS } \\
\mathrm{m} / \mathrm{z}\end{array}$ \\
\hline 8 & $\begin{array}{l}\left.\text { (In } \mathrm{CD}_{3} \mathrm{OD}\right): \\
7.19(2 \mathrm{H}, \mathrm{s}, \mathrm{H}-4,5), 8.02(1 \mathrm{H}, \\
\mathrm{s}, \mathrm{N}-\mathrm{H})\end{array}$ & $\begin{array}{l}135.9(\mathrm{C}-2), 121(\mathrm{C}-4,5) \\
\text { DEPT-135'- 121(C-4, } \\
\text { 5) }\end{array}$ & $\begin{array}{l}\text { ESI (+ve): } \\
\text { m/z: } 165\left[\mathrm{M}+\mathrm{NH}_{4}\right]^{+} \\
\text {ESI (-ve): } \\
\text { m/z: } 294[2 \mathrm{M}-\mathrm{H}]^{-}\end{array}$ \\
\hline 9 & $\begin{array}{l}\left(\mathrm{In} \mathrm{CDCl}_{3}\right) \text { : } \\
8.67(2 \mathrm{H}, \text { broad, } 2 \mathrm{~N}-\mathrm{H}), \\
8.37(2 \mathrm{H}, \mathrm{d}, \mathrm{J}=7.98 \mathrm{~Hz}, \mathrm{H}-4 \text { ', } \\
\text { 5'), 7.80(2H, dd, J=7.7 Hz, } \\
\text { 1.7 Hz, H-3, 6,), } 7.29(2 \mathrm{H}, \\
\text { ddd, J=7.7 Hz, } 7.3 \mathrm{~Hz}, 1.7 \\
\mathrm{~Hz}, \mathrm{H}-4,5)\end{array}$ & $\begin{array}{l}\text { 121.19, 123.23, 136.99, } \\
149.15,156.11\end{array}$ & - \\
\hline 10 & $\begin{array}{l}\left.\text { (In DMSO-d })_{6}\right) \\
9.13(2 \mathrm{H}, \text { broad, } 2 \mathrm{~N}-\mathrm{H}), 8.60 \\
\left(2 \mathrm{H}, \mathrm{d}, \mathrm{J}=8.1 \mathrm{~Hz}, \mathrm{H}-4^{\prime}, 5^{\prime}\right), \\
8.35(2 \mathrm{H}, \mathrm{dd}, \mathrm{J}=7.7 \mathrm{~Hz}, \mathrm{~J}=1.7 \\
\mathrm{Hz}, \mathrm{H}-3,6), 7.82(2 \mathrm{H}, \mathrm{ddd}, \\
\mathrm{J}=7.7 \mathrm{~Hz}, 7.3 \mathrm{~Hz}, 1.7 \mathrm{~Hz}, \mathrm{H}- \\
4,5)\end{array}$ & $\begin{array}{l}157\left(\mathrm{C}-2,2^{\prime}\right), 150(\mathrm{C}-3, \\
6), 140(\mathrm{C}-4), 127(\mathrm{C}-5), \\
124\left(\mathrm{C}-4^{\prime}, 5^{\prime}\right) \\
\text { DEPT-135-150 (C-3, } \\
6), 140\left(\mathrm{C}^{\circ} 4\right), 127(\mathrm{C}-5), \\
124\left(\mathrm{C}-4^{\prime}, 5^{\prime}\right)\end{array}$ & $\begin{array}{l}\text { ESI (+ve): m/z: } \\
360,362,364 \\
{[\mathrm{M}+\mathrm{Na}]^{+}}\end{array}$ \\
\hline
\end{tabular}


Table-2: Spectral data for compounds 11-12

\begin{tabular}{|c|c|c|c|}
\hline Compound & $\begin{array}{c}{ }^{1} \mathrm{H}-\mathrm{NMR} \\
\left(\mathrm{CDCl}_{3}\right),(\delta, \mathrm{ppm}) \\
\end{array}$ & $\begin{array}{c}{ }^{13} \mathrm{C}-\mathrm{NMR} \\
\left(\mathrm{CDCl}_{3}\right),(\delta, \mathrm{ppm})\end{array}$ & $\begin{array}{c}\text { MASS } \\
\mathrm{m} / \mathrm{z}\end{array}$ \\
\hline 11 & $\begin{array}{l}7.62 \quad\left(4 \mathrm{H}, \quad \mathrm{dd}, \mathrm{J}_{\mathrm{o}}=7.2 \mathrm{~Hz},\right. \\
\left.\mathrm{J}_{\mathrm{m}}=1.6 \mathrm{~Hz}, \mathrm{H}-2,2^{\prime}, 6,6^{\prime}\right), 7.46 \\
\left(4 \mathrm{H}, \mathrm{dd}, \mathrm{J}_{\mathrm{o}}=7.6 \mathrm{~Hz}, 7.2 \mathrm{~Hz},\right. \\
\left.\mathrm{H}-3,33^{\prime}, 5,5^{\prime}\right), 7.36(2 \mathrm{H}, \mathrm{dt}, \\
\left.\mathrm{J}_{\mathrm{o}}=7.6 \mathrm{~Hz}, \mathrm{~J}_{\mathrm{m}}=1.6 \mathrm{~Hz}, \mathrm{H}-4,4^{\prime}\right)\end{array}$ & $\begin{array}{l}141.20\left(\mathrm{C}-1,1^{\prime}\right), 128.72(\mathrm{C}- \\
\left.2,2^{\prime}, 6,6^{\prime}\right), 127.22\left(\mathrm{C}-4,4^{\prime}\right), \\
127.14\left(\mathrm{C}-3,3^{\prime}, 5,5^{\prime}\right) \\
\text { DEPT-135'-128.72 (C-2, 2', } \\
\left.6,6^{\prime}\right), 127.22\left(\mathrm{C}-4,4^{\prime}\right), \\
127.14\left(\mathrm{C}-3,3^{\prime}, 5,5^{\prime}\right)\end{array}$ & 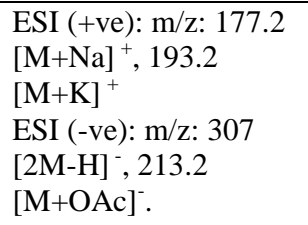 \\
\hline $12 a$ & $\begin{array}{l}7.57\left(2 \mathrm{H}, \mathrm{dd}, \mathrm{J}_{\mathrm{o}}=8 \mathrm{~Hz}, \mathrm{~J}_{\mathrm{m}}=1.2\right. \\
\left.\mathrm{Hz}, \mathrm{H}-2^{\prime}, 6^{\prime}\right), 7.48(2 \mathrm{H}, \mathrm{d}, \\
\left.\mathrm{J}_{\mathrm{o}}=7.6 \mathrm{~Hz}, \mathrm{H}-3,5\right), 7.41(2 \mathrm{H}, \\
\mathrm{dd}^{\prime}, \mathrm{J}_{\mathrm{o}}=8 \mathrm{~Hz}, \mathrm{~J}_{\mathrm{m}}=1.2 \mathrm{~Hz}, \mathrm{H}-3^{\prime}, \\
\left.5^{\prime}\right), 7.31\left(1 \mathrm{H}, \mathrm{t}, \mathrm{J}_{\mathrm{o}}=8.0 \mathrm{~Hz}, \mathrm{H}-\right. \\
\left.4^{\prime}\right), 7.24\left(2 \mathrm{H}, \mathrm{d}, \mathrm{J}_{\mathrm{o}}=7.6 \mathrm{~Hz}, \mathrm{H}-\right. \\
2,6), 2.38\left(3 \mathrm{H}, \mathrm{s}, \mathrm{CH}_{3}\right),\end{array}$ & 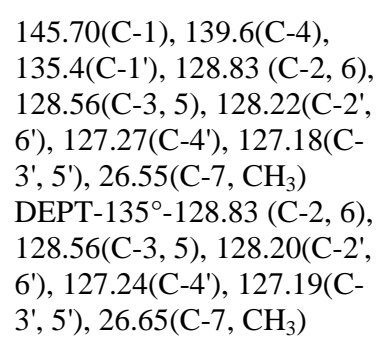 & 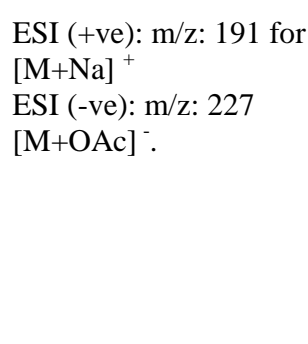 \\
\hline $12 \mathrm{~b}$ & $\begin{array}{l}8.02\left(2 \mathrm{H}, \mathrm{d}, \mathrm{J}_{\mathrm{o}}=8.4 \mathrm{~Hz}, \mathrm{H}-2,6\right) \\
\delta 7.68\left(2 \mathrm{H}, \mathrm{d}, \mathrm{J}_{\mathrm{o}}=8.4 \mathrm{~Hz}, \mathrm{H}-\right. \\
3,5), 7.63\left(2 \mathrm{H}, \mathrm{dd}, \mathrm{J}_{\mathrm{o}}=7.2 \mathrm{~Hz},\right. \\
\left.\mathrm{J}_{\mathrm{m}}=1.2 \mathrm{~Hz}, \mathrm{H}-2^{\prime}, 6^{\prime}\right), \delta 7.46 \\
\left(2 \mathrm{H}, \mathrm{dt}, \mathrm{J}_{\mathrm{o}}=7.2 \mathrm{~Hz}, \mathrm{~J}_{\mathrm{m}}=1.2 \mathrm{~Hz},\right. \\
\left.\mathrm{H}-3^{\prime}, 5^{\prime}\right), 7.39\left(1 \mathrm{H}, \mathrm{dt}, \mathrm{J}_{\mathrm{o}}=7.2\right. \\
\left.\mathrm{Hz}, \mathrm{J}_{\mathrm{m}}=1.2 \mathrm{~Hz}, \mathrm{H}-4^{\prime}\right), \delta 2.63 \\
\left(3 \mathrm{H}, \mathrm{s}, \mathrm{CH}_{3}\right)\end{array}$ & $\begin{array}{l}197.74(\mathrm{C}-7, \mathrm{C}=\mathrm{O}), \\
145.74(\mathrm{C}-1), 139.8(\mathrm{C}-4), \\
135.8\left(\mathrm{C}-1^{\prime}\right), 128.93(\mathrm{C}-3,5), \\
128.66(\mathrm{C}-2,6), 128.20\left(\mathrm{C}-2^{\prime},\right. \\
\left.6^{\prime}\right), 127.24\left(\mathrm{C}-4^{\prime}\right), 127.19(\mathrm{C}- \\
\left.3^{\prime}, 5^{\prime}\right), 26.65\left(\mathrm{C}-8, \mathrm{CH}_{3}\right) \\
\text { DEPT-135'-128.93(C-3, 5), } \\
128.66(\mathrm{C}-2,6), 128.20\left(\mathrm{C}-2^{\prime},\right. \\
\left.6^{\prime}\right), 127.24\left(\mathrm{C}-4^{\prime}\right), 127.19(\mathrm{C}- \\
\left.3^{\prime}, 5^{\prime}\right), 26.65\left(\mathrm{C}-8, \mathrm{CH}_{3}\right)\end{array}$ & $\begin{array}{l}\text { ESI (+ve): m/z: } \\
414[2 \mathrm{M}+\mathrm{Na}-\mathrm{H}]^{+}, \\
219.1[\mathrm{M}+\mathrm{Na}]^{+} \\
\text {ESI (-ve): m/z: } 255.3 \\
{\text { [M+OAc }]^{-}}\end{array}$ \\
\hline $12 \mathrm{c}$ & $\begin{array}{l}8.29\left(2 \mathrm{H}, \mathrm{d}, \mathrm{J}_{\mathrm{o}}=8.8 \mathrm{~Hz}, \mathrm{H}-2,\right. \\
6), 7.73\left(2 \mathrm{H}, \mathrm{d}, \mathrm{J}_{\mathrm{o}}=8.8 \mathrm{~Hz}, \mathrm{H}-\right. \\
3,5), 7.62\left(2 \mathrm{H}, \mathrm{dd}, \mathrm{J}_{\mathrm{o}}=7.2 \mathrm{~Hz},\right. \\
\left.\mathrm{J}_{\mathrm{m}}=1.6 \mathrm{~Hz}, \mathrm{H}-2^{\prime}, 6^{\prime}\right), 7.49(2 \mathrm{H}, \\
\mathrm{dd}^{\prime} \mathrm{J}_{\mathrm{o}}=7.2 \mathrm{~Hz}, 7.6 \mathrm{~Hz}, \mathrm{H}-3^{\prime}, \\
\left.5^{\prime}\right), 7.45\left(1 \mathrm{H}, \mathrm{dt}, \mathrm{J}_{\mathrm{o}}=7.6 \mathrm{~Hz},\right. \\
\left.\mathrm{J}_{\mathrm{m}}=1.6 \mathrm{~Hz}, \mathrm{H}-4^{\prime}\right) .\end{array}$ & $\begin{array}{l}147.59(\mathrm{C}-1), 147.02(\mathrm{C}-4), \\
138.72\left(\mathrm{C}-1^{\prime}\right), 129.12(\mathrm{C}-2, \\
6), 128.89(\mathrm{C}-3,5), 127.76 \\
\left(\mathrm{C}^{\prime} 2^{\prime}, 6^{\prime}\right), 127.37\left(\mathrm{C}^{\prime} 3^{\prime}, 5^{\prime}\right), \\
124.07\left(\mathrm{C}-4^{\prime}\right) \\
\text { DEPT-135'-129.12(C-2, 6), } \\
128.89(\mathrm{C}-3,5), 127.76,(\mathrm{C}- \\
\left.2^{\prime}, 6^{\prime}\right), 127.37\left(\mathrm{C}-3^{\prime}, 5^{\prime}\right), \\
124.07\left(\mathrm{C}-4^{\prime}\right)\end{array}$ & $\begin{array}{l}\text { ESI (+ve): m/z: } 222.2 \\
{[\mathrm{M}+\mathrm{Na}]^{+}} \\
\text {ESI (-ve): m/z: } \\
258.3[\mathrm{M}+\mathrm{OAc}]^{-}, 199 \\
{[\mathrm{M}]^{-}}\end{array}$ \\
\hline 12d & $\begin{array}{l}8.45\left(1 \mathrm{H}, \mathrm{dd},, \mathrm{J}_{\mathrm{m}}=1.6 \mathrm{~Hz}, 2\right. \\
\mathrm{Hz}, \mathrm{H}-2), 8.19(1 \mathrm{H}, \mathrm{dd}, \\
\left.\mathrm{J}_{\mathrm{o}}=8.0 \mathrm{~Hz}, \mathrm{~J}_{\mathrm{m}}=1.2 \mathrm{~Hz}, \mathrm{H}-6\right), \\
7.90\left(1 \mathrm{H}, \mathrm{dd}, \mathrm{J}_{\mathrm{o}}=7.6 \mathrm{~Hz},\right. \\
\left.\mathrm{J}_{\mathrm{m}}=1.2 \mathrm{~Hz}, \mathrm{H}-4\right), 7.58-7.63 \\
\left(3 \mathrm{H}, \mathrm{m}, \mathrm{H}-5 \& \mathrm{H}-2^{\prime}, 6^{\prime}\right), 7.49 \\
\left(2 \mathrm{H}, \mathrm{t}, \mathrm{J}_{\mathrm{o}}=7.2 \mathrm{~Hz}, \mathrm{H}^{\prime} 3^{\prime}, 5^{\prime}\right), \\
7.43\left(1 \mathrm{H}, \mathrm{dt}, \mathrm{J}_{\mathrm{o}}=7.2 \mathrm{~Hz},\right. \\
\left.\mathrm{J}_{\mathrm{m}}=1.2 \mathrm{~Hz}, \mathrm{H}-4^{\prime}\right) .\end{array}$ & $\begin{array}{l}148(\mathrm{C}-1), 142(\mathrm{C}-3), 138 \\
\left(\mathrm{C}-1^{\prime}\right), 133(\mathrm{C}-2), 129.67(\mathrm{C}- \\
6), 129.12(\mathrm{C}-4), 128.50(\mathrm{C}- \\
5), 127.77\left(\mathrm{C}-2^{\prime}, 6^{\prime}\right), \\
121.99\left(\mathrm{C}-3^{\prime}, 5^{\prime}\right), 121.91(\mathrm{C}- \\
\left.4^{\prime}\right) \\
\text { DEPT-135'-133(C-2), } \\
129.67(\mathrm{C}-6), 129.12(\mathrm{C}-4), \\
128.50(\mathrm{C}-5), 127.77\left(\mathrm{C}-2^{\prime},\right. \\
\left.6^{\prime}\right), 121.99\left(\mathrm{C}-3^{\prime}, 5^{\prime}\right), \\
121.91\left(\mathrm{C}-4^{\prime}\right)\end{array}$ & $\begin{array}{l}\text { ESI }(+v e): m / z: 222.2 \\
{\left[^{M}+N a\right]^{+}} \\
\text {ESI (-ve): m/z: } \\
\text { 258.3[M+OAc] }{ }^{-}\end{array}$ \\
\hline
\end{tabular}




\begin{tabular}{|c|c|c|c|}
\hline Compound & $\begin{array}{c}{ }^{1} \mathrm{H}-\mathrm{NMR} \\
\left(\mathrm{CDCl}_{3}\right),(\delta, \mathrm{ppm})\end{array}$ & $\begin{array}{c}{ }^{13} \mathrm{C}-\mathrm{NMR} \\
\left(\mathrm{CDCl}_{3}\right),(\delta, \mathrm{ppm})\end{array}$ & $\begin{array}{c}\text { MASS } \\
\mathrm{m} / \mathrm{z}\end{array}$ \\
\hline $12 \mathrm{e}$ & $\begin{array}{l}\text { 7.50-7.62 (4H, m, H-2, 2', 6, } \\
\left.6^{\prime}\right), 7.43\left(2 \mathrm{H}, \mathrm{dd}, \mathrm{J}_{\mathrm{o}}=7.2 \mathrm{~Hz},\right. \\
\left.7.5 \mathrm{~Hz}, \mathrm{H}-3^{\prime}, 5^{\prime}\right), 7.33(1 \mathrm{H}, \mathrm{dt}, \\
\left.\mathrm{J}_{\mathrm{o}}=7.5 \mathrm{~Hz}, \mathrm{H}^{\prime} 4^{\prime}\right), 7.12(2 \mathrm{H}, \mathrm{dt}, \\
\left.\mathrm{J}_{\mathrm{o}}=8.7 \mathrm{~Hz}, \mathrm{~J}_{\mathrm{m}}=2.1 \mathrm{~Hz}, \mathrm{H}-3,5\right)\end{array}$ & $\begin{array}{l}164(\mathrm{C}-1), 160.80(\mathrm{C}-4), \\
141.22\left(\mathrm{C}-1^{\prime}\right), 138.97(\mathrm{C}-2, \\
6), 137.32(\mathrm{C}-3,5), 137.28 \\
\left(\mathrm{C}-2^{\prime}, 6^{\prime}\right), 128.79\left(\mathrm{C}-3^{\prime}, 5^{\prime}\right), \\
115.72(\mathrm{C}-2,6) \\
\text { DEPT-135'-128.97 (C-2, } \\
6), 137.32(\mathrm{C}-3,5), 137.28 \\
\left(\mathrm{C}^{\circ} 2^{\prime}, 6^{\prime}\right), 128.79\left(\mathrm{C}-3^{\prime}, 5^{\prime}\right), \\
115.72(\mathrm{C}-2,6)\end{array}$ & $\begin{array}{l}\text { ESI (+ve): m/z: } \\
193[\mathrm{M}+\mathrm{Na}]^{+} \\
\text {ESI (-ve): m/z: } \\
228.4[\mathrm{M}+\mathrm{OAc}-\mathrm{H}]^{-}\end{array}$ \\
\hline $12 f$ & $\begin{array}{l}7.68(4 \mathrm{H}, \mathrm{s}(\mathrm{br}), \mathrm{H}-2,3,5,6) \\
7.58 \quad\left(2 \mathrm{H}, \mathrm{dt}, \quad \mathrm{J}_{\mathrm{o}}=6.9 \mathrm{~Hz},\right. \\
\left.\mathrm{J}_{\mathrm{m}}=1.8 \mathrm{~Hz}, \mathrm{H}-2^{\prime}, 6^{\prime}\right), 7.46(2 \mathrm{H}, \\
\mathrm{dt}, \mathrm{J}_{\mathrm{o}}=6.9 \mathrm{~Hz}, \mathrm{~J}_{\mathrm{m}}=1.8 \mathrm{~Hz}, \mathrm{H}-3^{\prime}, \\
\left.5^{\prime}\right), 7.39\left(1 \mathrm{H}, \mathrm{dt}, \mathrm{J}_{\mathrm{o}}=7.2 \mathrm{~Hz},\right. \\
\left.\mathrm{J}_{\mathrm{m}}=1.8 \mathrm{~Hz}, \mathrm{H}^{\prime}-4^{\prime}\right) .\end{array}$ & 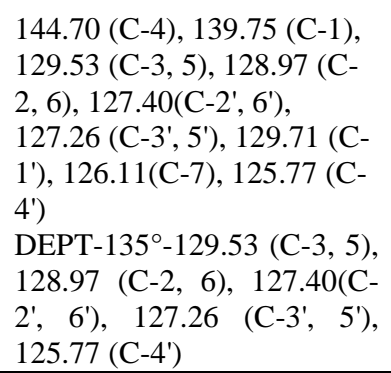 & $\begin{array}{l}\text { ESI (+ve): m/z: } 242.3 \\
{[\mathrm{M}+\mathrm{Na}]^{+}} \\
\text {ESI }(-\mathrm{ve}): \mathrm{m} / \mathrm{z}: \\
253.5\left[\mathrm{M}+{ }^{35} \mathrm{Cl}\right]^{-} \\
255.5\left[\mathrm{M}+{ }^{37} \mathrm{Cl}\right]^{-}\end{array}$ \\
\hline
\end{tabular}

Table-3: Spectral data for compounds 13

\begin{tabular}{|c|c|c|c|}
\hline Compound & $\begin{array}{c}{ }^{\mathrm{I}} \mathrm{H}-\mathrm{NMR} \\
\left(\mathrm{CDCl}_{3}\right),(\delta, \mathrm{ppm})\end{array}$ & $\begin{array}{c}{ }^{13} \mathrm{C}-\mathrm{NMR} \\
\left(\mathrm{CDCl}_{3}\right),(\delta, \mathrm{ppm})\end{array}$ & $\begin{array}{c}\text { Mass } \\
\mathrm{m} / \mathrm{z}\end{array}$ \\
\hline $13 a^{*}$ & $\begin{array}{l}7.81\left(1 \mathrm{H}, \mathrm{t}, \mathrm{J}_{\mathrm{m}}=1.8 \mathrm{~Hz}, \mathrm{H}-2\right), 7.65 \\
\left(4 \mathrm{H}, \mathrm{dd}, \mathrm{J}_{\mathrm{o}}=6.9 \mathrm{~Hz}, \mathrm{~J}_{\mathrm{m}}=2.1 \mathrm{~Hz}, \mathrm{H}-\right. \\
\left.2^{\prime}, 2^{\prime \prime}, 6,6 "\right), 7.59(2 \mathrm{H}, \mathrm{m}, \mathrm{H}-4,6), \\
7.52(1 \mathrm{H}, \mathrm{dd}, \mathrm{J}=6.0 \mathrm{~Hz}, 5.4 \mathrm{~Hz}, \mathrm{H}-5), \\
7.48(4 \mathrm{H}, \mathrm{m}, \mathrm{H}-3 \text { ' }, 3 ", 5 ', 5 "), 7.37 \\
\left(2 \mathrm{H}, \mathrm{dt}, \mathrm{J}_{\mathrm{o}}=7.1 \mathrm{~Hz}, \mathrm{~J}_{\mathrm{m}}=2.1 \mathrm{~Hz}, \mathrm{H}-4 \text { ', }\right. \\
\left.4^{\prime \prime}\right)\end{array}$ & - & - \\
\hline $13 b$ & $\begin{array}{l}7.63\left(4 \mathrm{H}, \mathrm{dd}, \mathrm{J}_{\mathrm{o}}=6.9 \mathrm{~Hz}, \mathrm{~J}_{\mathrm{m}}=1.5 \mathrm{~Hz},\right. \\
\mathrm{H}-2,3,5,6), 7.55\left(4 \mathrm{H}, \mathrm{dt}, \mathrm{J}_{\mathrm{o}}=7.2 \mathrm{~Hz},\right. \\
\left.\mathrm{J}_{\mathrm{m}}=1.2 \mathrm{~Hz}, \mathrm{H}-2^{\prime}, 2^{\prime \prime}, 6^{\prime}, 6^{\prime \prime}\right), 7.44 \\
\left(4 \mathrm{H}, \mathrm{dd}, \mathrm{J}_{\mathrm{o}}=7.5 \mathrm{~Hz}, 7.2 \mathrm{~Hz}, \mathrm{H}-3^{\prime}, 3^{\prime \prime},\right. \\
\left.5^{\prime}, 5^{\prime \prime}\right), 7.35\left(2 \mathrm{H}, \mathrm{dt}, \mathrm{J}_{\mathrm{o}}=7.5 \mathrm{~Hz},\right. \\
\left.\mathrm{J}_{\mathrm{m}}=1.2 \mathrm{~Hz}, \mathrm{H}-4^{\prime}, 4^{\prime \prime}\right) \\
8.05(1 \mathrm{H}, \mathrm{s}, \mathrm{H}-1) .92(1 \mathrm{H}, \mathrm{d}, \mathrm{J}=8.4\end{array}$ & 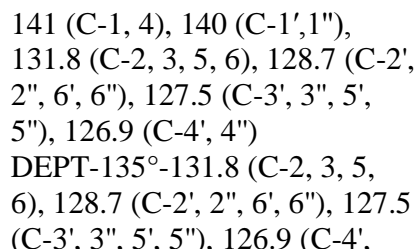 & $\begin{array}{l}\text { ESI (+ve): m/z: } \\
253.1[\mathrm{M}+\mathrm{Na}]^{+} \\
\text {ESI }(-\mathrm{ve}): \mathrm{m} / \mathrm{z}: \\
265.1\left[\mathrm{M}+{ }^{35} \mathrm{Cl}\right]^{-}, \\
228.4[\mathrm{M}-2 \mathrm{H}]^{2-} \\
\text { ESI (+ve): } \mathrm{m} / \mathrm{z} \text { : }\end{array}$ \\
\hline $13 c$ & $\begin{array}{l}\mathrm{Hz}, \mathrm{H}-3), 7.90\left(1 \mathrm{H}, \mathrm{d}, \mathrm{J}_{\mathrm{o}}=8.4 \mathrm{~Hz}, \mathrm{H}-\right. \\
4), 7.89\left(1 \mathrm{H}, \mathrm{dd}, \mathrm{J}_{\mathrm{o}}=6.8 \mathrm{~Hz}, 7.2 \mathrm{~Hz},\right. \\
\mathrm{H}-8), 7.88\left(1 \mathrm{H}, \mathrm{d}, \mathrm{J}_{\mathrm{o}}=6.8 \mathrm{~Hz}, \mathrm{H}-7\right) \text {, } \\
7.71-7.77(3 \mathrm{H}, \mathrm{m}, \mathrm{H}-5,6,7), 7.46- \\
7.54\left(4 \mathrm{H}, \mathrm{m}, \mathrm{H}-2^{\prime}, 3^{\prime}, 5^{\prime}, 6^{\prime}\right), 7.39 \\
\left(1 \mathrm{H}, \mathrm{dt}, \mathrm{J}_{\mathrm{o}}=6.8 \mathrm{~Hz}, 7.2 \mathrm{~Hz}, \mathrm{H}-4^{\prime}\right) .\end{array}$ & $\begin{array}{l}\left.4^{\prime \prime}\right) \\
141.1(\mathrm{C}-2), 138.53\left(\mathrm{C}-1^{\prime}\right), \\
133.64(\mathrm{C}-9), 132.58(\mathrm{C}-10), \\
\text { 128.84, 128.39, 128.17, 127.62, } \\
\text { 127.41, 127.33, 126.27, 125.91, } \\
\text { 125.78, 125.58 } \\
\text { DEPT-135'-128.84, 128.39, } \\
\text { 128.17, 127.62, 127.41, 127.33, } \\
126.27,125.91,125.78,125.58\end{array}$ & $\begin{array}{l}247[\mathrm{M}+42+\mathrm{H}]^{+} \\
\text {ESI (-ve): } \\
\text { m/z: } 206[\mathrm{M}-\mathrm{H}]^{-}\end{array}$ \\
\hline
\end{tabular}




\section{Results and Discussion}

The Pd catalyst was easily prepared from N-(2'-imidazolyl)-2- aminopyridine by the reaction with $\mathrm{Na}_{2} \mathrm{PdCl}_{4}$ in $\mathrm{MeOH}$ at room temperature (Scheme 2). Complex 10 was obtained with high purity as orange-yellow powder.

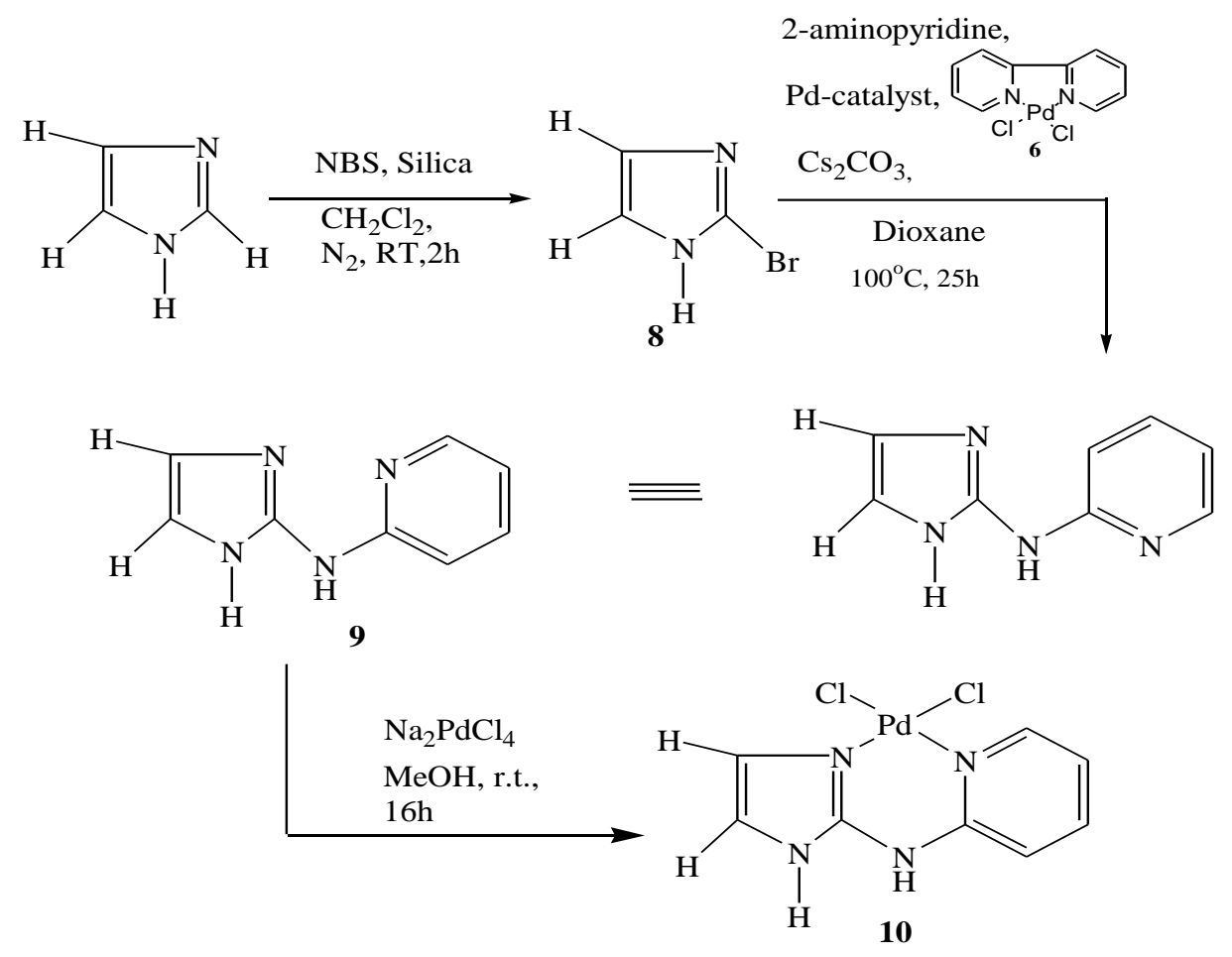

Scheme 2: Synthesis of Pd complex 10

The Pd-complex 10 was characterized from the ${ }^{1} \mathrm{H}$ NMR, ${ }^{13} \mathrm{C}-\mathrm{NMR}$ and Mass spectral data analysis (table 1). In the complex $\mathbf{1 0}$ each of the protons shifts to downfield by about 0.3-0.6 ppm than that of the ligand indicating the complex formation. The molecular mass for $\left[\mathrm{M}+\mathrm{Na}^{+}\right]$confirms the molecular structure of $\mathbf{1 0}$ with dichloro patern (peak ratio 9: 6: 1).

With the Pd catalyst 10, we tested the Suzuki-Miyaura coupling to obtain the product 1,1biphenyl, 11 from the reaction of different halobenzenes (Scheme 3, table-4, entry 1, 2, 3) with phenylboronic acid in the presence of $\mathrm{K}_{2} \mathrm{CO}_{3}$. The reaction was done in toluene at $100-105^{\circ} \mathrm{C}$. 


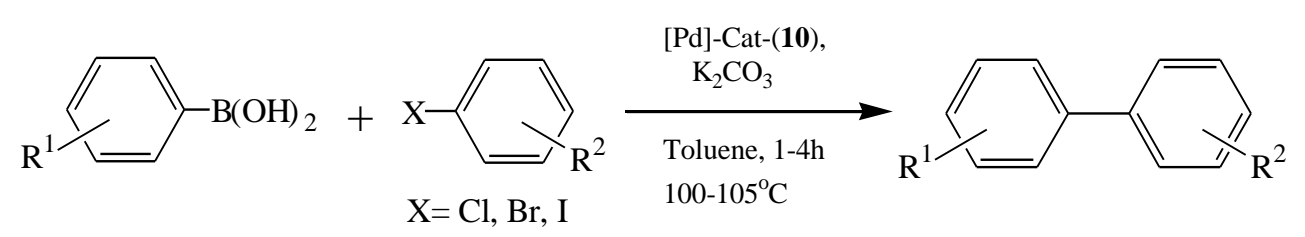

Scheme 3

All the three reactions resulted in the formation of the same compound 1, 1'- biphenyl, 11 having melting point $68-70^{\circ} \mathrm{C}$ with yields of $34 \%, 97 \%$ and $90 \%$ with chloro, bromo and iodo benzene respectively. The reaction of chlorobenzene is most time consuming and the yield is very poor. To facilitate the reaction, the amount Pd [cat] needed is higher than the other two reactions.

Table 4: Suzuki-Miyaura coupling of aryl halides with arylboronic acid to synthesize 1, 1'- biphenyl, 11

\begin{tabular}{|c|c|c|c|c|c|c|}
\hline Entry & $\begin{array}{c}\text { Arylboronic acid } \\
\mathrm{R}^{1}=\text { Substituent }\end{array}$ & $\begin{array}{c}\text { Aryl halides } \\
\left(\mathrm{R}^{2}=\mathrm{H}\right) \\
\mathrm{X}\end{array}$ & $\mathrm{T}\left({ }^{\circ} \mathrm{C}\right)$ & $\begin{array}{c}\mathbf{6}(\mathrm{mmole} \\
\%)\end{array}$ & Time (h) & $\begin{array}{c}\text { Yield } \\
\text { (Product } \\
\mathbf{1 1})(\%)\end{array}$ \\
\hline 1 & $\mathrm{H}$ & $\mathrm{Cl}$ & 105 & 3 & 12 & 34 \\
2 & $\mathrm{H}$ & $\mathrm{Br}$ & 105 & 1 & 2 & 97 \\
3 & $\mathrm{H}$ & $\mathrm{I}$ & 105 & 1 & 1.5 & 90 \\
\hline
\end{tabular}

Then we tested the Suzuki-Miyaura (SM) coupling reaction of 4-Bromotoluene with phenylboronic acid in the presence of $\mathrm{K}_{2} \mathrm{CO}_{3}$ as a model reaction. The reaction was done in toluene at $100-105^{\circ} \mathrm{C}$ with $1 \mathrm{mmol} \%$ of $\mathbf{1 0}$. The reaction led to $97 \%$ of the product, 4-methylbiphenyl, 12a in $2 \mathrm{hrs}$. and all of the starting material was converted into the product (Scheme 3, table 5, entry $\mathbf{1}$ ). We repeated the SM coupling reaction of phenyl boronic acid with electron withdrawing substituents $\left(-\mathrm{COCH}_{3},-\mathrm{NO}_{2} \& \mathrm{~F}\right)$ in bromides (table 5, entry 2, 3, $4 \& 5$ ), the coupling was very easy and the yields were excellent. The catalyst $\mathbf{1 0}$ was also used for the coupling of phenyl boronic acid with 4 triflouromethyliodobenzene (table $\mathbf{5}$, entry $\mathbf{6}$ ), the product, $\mathbf{1 2 f}$ was obtained in excellent yield.

We also tested the catalyst, 10, with the dibromides and naphthylbromides.The coupling with dibromides led to the production of terphenyls, 13a and 13b in good yield (table $\mathbf{6}$, entry $1 \& 2$ ). The naphthylbromide gave phenylnaphthalene, 13c (table 6, entry 3), in quantitative yield.

From the above results we found that Pd complex 10 showed outstanding performance. The complex 10 was very stable to oxygen and moisture: no change in its activity was observed, when it was exposed to air or water. 
Table 5: Suzuki-Miyaura coupling of substituted aryl halides with arylboronic acid

\begin{tabular}{|c|c|c|c|c|c|c|c|}
\hline Entry & $\begin{array}{c}\text { Arylboronic } \\
\text { acid, } \\
\mathrm{R}^{1}\end{array}$ & $\begin{array}{c}\text { Aryl halides } \\
\mathrm{R}^{2}=\text { Substituent } \\
\mathrm{X}=\mathrm{Br}, \mathrm{I}\end{array}$ & $\mathrm{T}\left({ }^{\circ} \mathrm{C}\right)$ & $\begin{array}{c}\mathbf{6}(\mathrm{mmole} \\
\%)\end{array}$ & Time (h) & $\begin{array}{c}\text { Product } \\
\mathbf{1 2}\end{array}$ & $\begin{array}{c}\text { Yield } \\
(\%)\end{array}$ \\
\hline 1 & $\mathrm{H}$ & $4-\mathrm{Me}$ & 105 & 1 & 2 & $\mathrm{a}$ & 97 \\
2 & $\mathrm{H}$ & $4-\mathrm{COCH}_{3}$ & 105 & 1 & 2 & $\mathrm{~b}$ & 91 \\
3 & $\mathrm{H}$ & $4-\mathrm{NO}_{2}$ & 105 & 1 & 1.5 & $\mathrm{c}$ & 100 \\
4 & $\mathrm{H}$ & $3-\mathrm{NO}_{2}$ & 105 & 1 & 1.5 & $\mathrm{~d}$ & 100 \\
5 & $\mathrm{H}$ & $4-\mathrm{F}$ & 105 & 1 & 3.5 & $\mathrm{e}$ & 96 \\
6 & $\mathrm{H}$ & $4-\mathrm{CF}_{3}$ & 105 & 1 & 1.5 & $\mathrm{f}$ & 91 \\
\hline
\end{tabular}

Table 6: SM coupling of aryldibromides with phenyl boronic acid in presence of complex 10

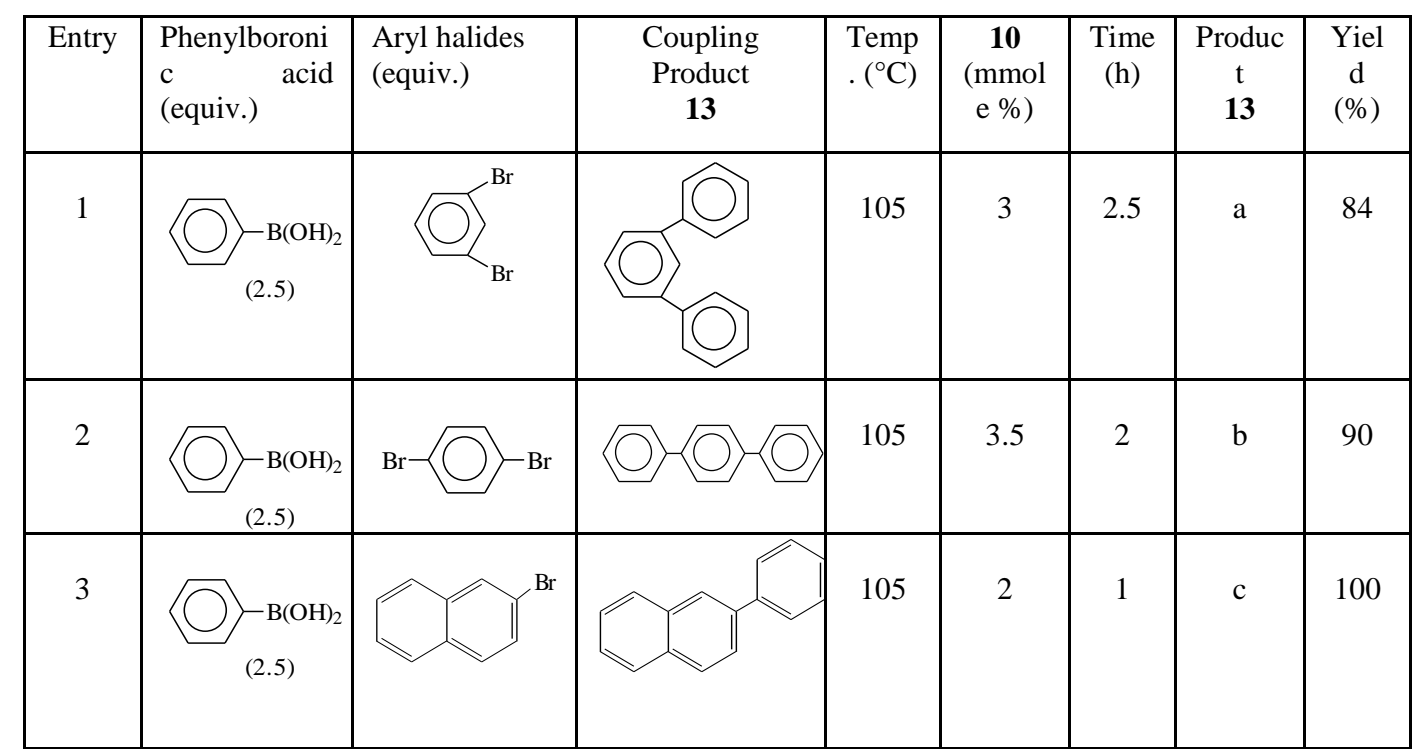


The plausible mechanism for the Suzuki coupling reaction of aryl boronic acids with aryl halides can be represented according to the literature ${ }^{22-25}$ as shown below:

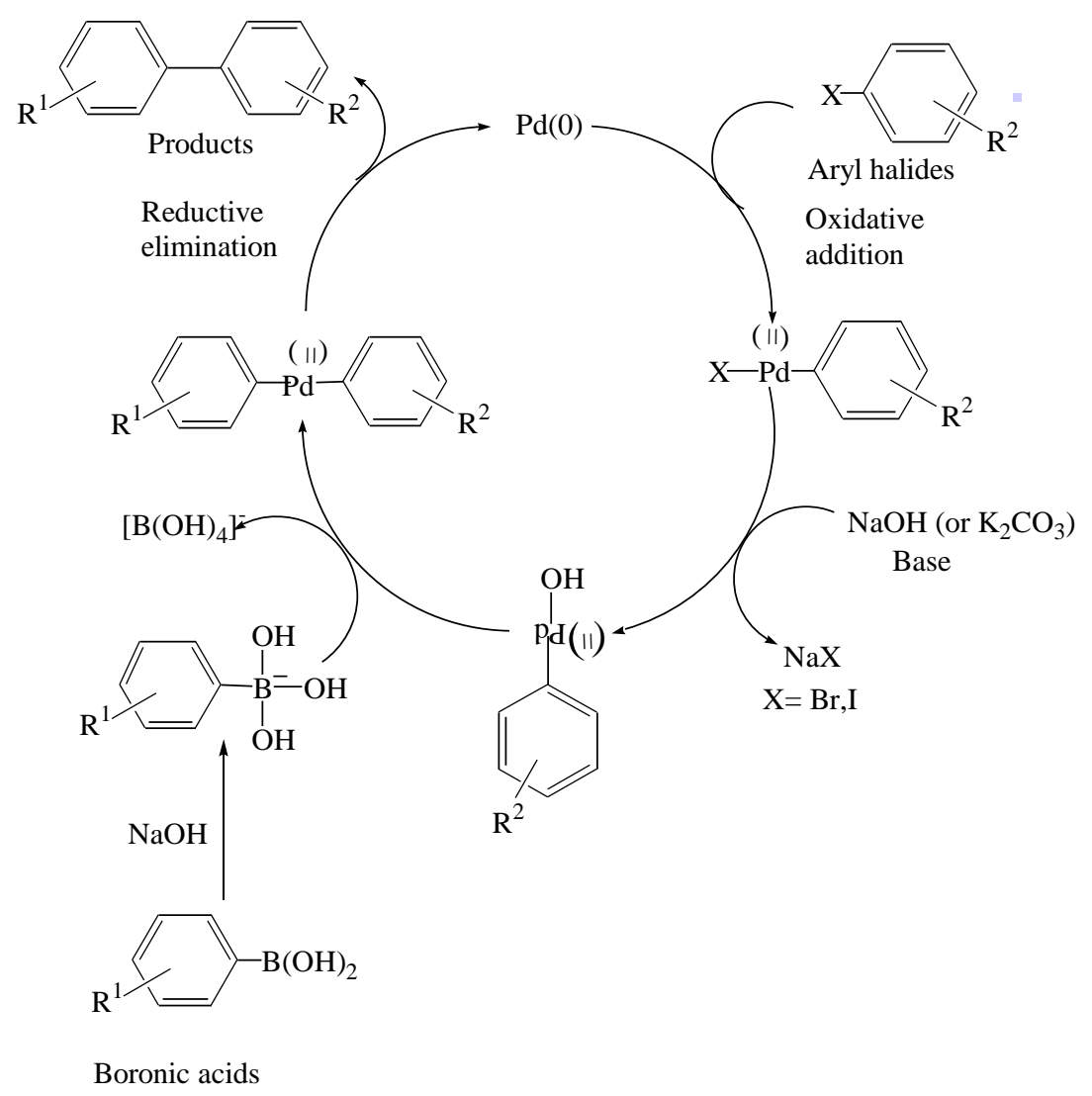

Scheme 4: The mechanism of Suzuki-Miyaura cross coupling reaction.

\section{Conclusions}

On the basis of the reaction time, yield, and mechanistic pathway it was observed that the aryl halides substituted with electron withdrawing groups at para position were more reactive and had higher yield than the aryl halides substituted with electron donating group. Due to crowding effect aryl halides substituted with electron withdrawing groups at ortho position were less reactive to the coupling reaction than the para derivative.

In case of aryl halides the reaction of chloro derivatives were more time consuming and the yield was very poor. To facilitate the reaction, the amount [Pd] catalyst needed was higher than the bromo and iodo derivatives. 
We have shown in the above results and discussion that the Pd- complex $\mathbf{1 0}$ is highly efficient catalyst in Suzuki-Miyaura coupling reactions. It is very stable in various reaction conditions.

\section{Acknowledgement}

The authors express their thanks to Prof. Dr. L. Wessjohann \& Dr. L. Katrin Franke, Leibniez Institute of Plant Bio-chemistry, Halle (Saale), Germany and Prof .Tahsin J. Chow \& Mr. Minarul Islam, Academia Sinica, Nankang, Taipei, Taiwan, Rebublic of China, and BCSIR, Dhaka for giving instrumental help in recording the NMR and mass spectra.

\section{References}

1. J. Dupont, N. Beydoun, M. Pfeffer, J. Chem. Soc. Dalton Trans., 1989,1715.

2. J. Dupont, N. R. Basso, M. R. Meneghetti, R. A. Konrath, R. Burrow, M. Horner, Organometallics, 1997, 16, 2386.

3. (a) R. D.Osullivan, A. W. Parkins, J. Chem. Commun., 1984, 1165.

(b) J. Vicevte, I. Saurallamas. P. G. Jones, J. Chem. Soc. Dalton Trans, 1993, 3619.

4. (a) R.Vanhelder, G. Verberg, Recl. Trav. Chim. Pays-Bus, 1965, 84, 1263.

(b) I. R. Girling, D.A. Widdowson, Tetrahedron Lett., 1982, 23, 1957.

5. A. Kasahara, Bull. Chem. Soc. Jpn., 1968, 41, 1272.

6. E.C. Constable, A. M. W. C. Thompson, T. A. Leese, D. G. F. Reese, D. A. Tocher, Inorg. Chem. Acta.,1991, 182, 93.

7. H. Alper, J. Organomet., 1973, 61, 62.

8. N. D. Cameron, M. Kilner, J. Chem. Soc. Chem. Commun., 1975, 687.

9. H. Horino, N. Inoue, J. Org. Chem., 1981, 46, 4416.

10. V. S. Goncharov, E. E. Sirotina, Izv. Sib. Otd. Akad. Nauk. SSSR, Ser. Khim. Nauk, 986, , 3, 77.

11. J. Dehand, A. Mauro, H. Ossar, M. Peffer, R. H. D. Santos, J. R. Lechat, J. Organomet. Chem., 1983, 250, 537.

12. For selected reviews on SM cross-coupling, see:

(a) A. Suzuki, H. C. Brown, Organic Synthesis via Boranes, Vol.3, Aldrich Chemical Company. Inc., Milwaukee, USA, 2003; (b) N. Miyaura, A. Suzuki, Chem. Rev. 1995, 95. 2457; (c) S. Kotha, K. Lahiri, D. Kashinath, Tetrahedron, 2002, 58, 9633; (d) M. MorenoManas, R. Pleixats, R. M. Sebastian, A. Vallribera, A. Roglans, J. Organomet. Chem. 2004, 689, 3669; (e) K. C. Nicotaou, P. G. Bulger, D. Sarlah, Angew. Chem. 2005, 117, 4516; Angew. Chem. Int. Ed. 2005, 44, 4442; (f) S. Kotha, M. Behera, V. R. Shah. Synlett. 2005, 1877; (g) S. Kotha, K. Lahiri, Eur. J. Org. Chem. 2007, 1221; (h) H. Dovcet, Eur. J. Org. Chem. 2008, 12, 2013.

13. Reviews of cross-coupling methodology, see: 
(a) B. V. W. Maes, Top. Heterocycl. Chem. 2006, 1, 155; (b) S. Schroeter, C. Stock, T. Bach, Tetrahedron, 2005, 61, 2245; (c) Metal-catalyzed cross-coupling reactions (Eds: A. de Meijere, F. Diederich), Wiley-VCH, Weinheim, 2004; (d) J. Hassan, M. Sevignon, C. Gozzi, E. Schultz, M. Lemaire, Chem. Rev. 2002, 102, 1359; (e) A. Zapf, M. Beller, Chem. Commun, 2005, 431; (f) S. P. Stanforth, Tetrahedron, 1998, 54, 263.

14. (a) O. Navarro, R. A. Kelly, S. P. Nolan, J. Am. Chem. Soc. 2003, 125, 16194; (b) M. J. Dai, B. Liang, C. H. Wang, Z. J. You, J. Xiang. G. B. Dong, J. H, Chen, Z. Yang, Adv. Synth. Catal. 2004, 346, 1664; (c) G. Lu, R. Franzen, Q. Zhang, Y. J. Xu, Tetrahedron Lett. 2005, 46, 4255; (d) T. Mino, Y. Shirae, M. Sakamoto, T. Fujita, J. Org. Chem. 2005, 70, 2191; (e) A. Mukherjee, A. Sarker, Tetrahedron Lett, 2004, 45, 15; (f) G. R. Rosa, C. H. Rosa, F. Rominger, J. Dupont, A. L. Monteiro, Inorg. Chim. I Acta. 2006, 359, 1947.

15. (a) R. B. Bedford, Chem. Commun. 2003, 1787; (b) J. J. Singleton, Tetrahedron, 2003, 59, 1837.

16. T. Frenzel, W. Solodenko, A. Kirschning, A solid- phase bound catalysts-properties and applications. In M. R. Buchmeiser (Ed.) Polymeric Materials in organic synthesis and catalysis, Wiley- VCH, Weinheim, 2003, 201.

17. C. Najera, J. Gil- Motto, S. Karlstrom, L. R. Falvello, Org. Lett. 2005, 5, 1451.

18. W. Soledenko, C. Brochwitz, R. Wartchow, M. A Hashem, K. M. Dawood, M.Vaultier, A. Kirschning, Molecular Diversity, 2005, 9, 333.

19. D - H. Lee, J - Y. Jung, I - M. Lee, M- J. Jin, Eur. J. Org. Chem. 2008, 2, 356.

20. A, Kirschning C. Altwicker, G. Dräger, J. Harders, N. Hoffmann, U Hoffmann, H. Schönfeld, W. Solodenko, U. Kunz, Angew Chem, Int Ed.; 2001, 40, 3995.

21. M. A. Hashem, M. L. Bhowmik, A. Sultana, Journal of Bangladesh Chemical Soceity, 2011, 24(1), 70 .

22. K. Matos, J. A. Soderquist, J. Org. Chem., 1998, 63, 461.

23. J. K. Stille, K. S. Y. Lau, Acc. Chem. Res., 1977, 10, 434.

24. A. L. Casado, P. Espinet, Organometallics, 1998, 17, 954.

25. B. H. Ridgway, K. A. Woerpel, J. Org. Chem., 1998, 63, 458. 


\section{Graphical Abstract}

Synthesis of N-(2'-imidazolyl)-2aminopyridyl palladium dichloride and its application as catalyst in Suzuki-Miyaura cross coupling reaction.

Md. Abul Hashem* and Abeda Sultana
N-(2'-imidazolyl)-2- aminopyridyl palladium dichloride complex, $\mathbf{1 0}$ by Suzuki-Miyaura cross coupling led to the isolation of several substituted biaryls, triaryls and heterobiaryls derivatives by the reaction of substituted phenylboronic acids and substituted aryl chlorides, bromides and iodides in excellent yield.

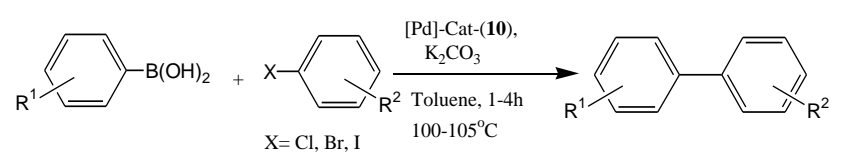

$\mathrm{R}^{1}=\mathrm{H}, \mathrm{X}=\mathrm{Cl}, \mathrm{Br}$, I

$\mathrm{R}^{2}=\mathrm{Me}, \mathrm{NO}_{2}, \mathrm{Br}, \mathrm{F}, \mathrm{COCH}_{3}, \mathrm{CF}_{3}$ etc. 